\title{
Panniculitis in childhood-onset systemic lupus erythematosus: a multicentric cohort study
}

\author{
Mônica Verdier ${ }^{1}$, Pedro Anuardo ${ }^{1}$, Natali Weniger Spelling Gormezano ${ }^{1,2}$, Ricardo Romiti ${ }^{3}$, \\ Lucia Maria Arruda Campos ${ }^{1}$, Nadia Emi Aikawa², Rosa Maria Rodrigues Pereira², Maria Teresa Terreri ${ }^{4}$, \\ Claudia Saad Magalhães ${ }^{5}$, Juliana C. O. A. Ferreira', Marco Felipe Castro Silva', Mariana Ferriani ${ }^{1}$, \\ Ana Paula Sakamoto ${ }^{4}$, Virginia Paes Leme Ferriani ${ }^{6}$, Maraísa Centeville ${ }^{7}$, Juliana Sato ${ }^{5}$, Maria Carolina Santos ${ }^{8}$, \\ Eloisa Bonfá ${ }^{2 \dagger}$ and Clovis Artur Silva ${ }^{1,2^{*}+}$ (i)
}

\begin{abstract}
Objective: To evaluate prevalence, clinical manifestations, laboratory abnormalities, treatment and outcome in a multicenter cohort of childhood-onset systemic lupus erythematosus (CSLE) patients with and without panniculitis.

Methods: Panniculitis was diagnosed due to painful subcutaneous nodules and/or plaques in deep dermis/subcutaneous tissues and lobular/mixed panniculitis with lymphocytic lobular inflammatory infiltrate in skin biopsy. Statistical analysis was performed using Bonferroni correction $(p<0.004)$.

Results: Panniculitis was observed in 6/847(0.7\%) CSLE. Painful subcutaneous erythematosus and indurated nodules were observed in 6/6 panniculitis patients and painful subcutaneous plaques in 4/6. Generalized distribution was evidenced in 3/ 6 and localized in upper limbs in 2/6 and face in 1/6. Cutaneous hyperpigmentation and/or cutaneous atrophy occurred in 5/6. Histopathology features showed lobular panniculitis without vasculitis in 5/6(one of them had concomitant obliterative vasculopathy due to antiphospholipid syndrome) and panniculitis with vasculitis in 1/6. Comparison between cSLE with panniculitis and 60 CSLE without panniculitis with same disease duration [2.75(0-11.4) vs. 2.83(0-11.8) years, $p=0.297]$, showed higher frequencies of constitutional involvement ( $67 \%$ vs. $10 \%, p=0.003)$ and leukopenia $(67 \%$ vs. $7 \%, p=0.002)$. Cutaneous atrophy and hyperpigmentation occurred in $83 \%$ of patients.
\end{abstract}

Conclusions: Panniculitis is a rare skin manifestation of CSLE occurring in the first three years of disease with considerable sequelae. The majority of patients have concomitant mild lupus manifestations.

Keywords: Lupus erythematosus panniculits, Childhood, Systemic lupus erythematosus and multicenter study

\section{Introduction}

Systemic lupus erythematosus (SLE) is an autoimmune disease that affects multiple organs and systems. Mucocutaneous involvement was described as initial manifestation in up to $81 \%$ childhood-onset SLE (cSLE) patients and in

\footnotetext{
* Correspondence: clovis.silva@hc.fm.usp.br

†Eloisa Bonfá and Clovis Artur Silva contributed equally to this work.

'Pediatric Rheumatology Unit, Children's Institute, Hospital das Clinicas HCFMUSP, Faculdade de Medicina, Universidade de Sao Paulo, Sao Paulo, SP, BR, Brazil

${ }^{2}$ Division of Rheumatology, Hospital das Clinicas HCFMUSP, Faculdade de Medicina, Universidade de Sao Paulo, Av. Dr. Eneas Carvalho Aguiar, 647 Cerqueira César, São Paulo, SP 05403-000, Brazil

Full list of author information is available at the end of the article
}

up to $100 \%$ of them during the disease course [1-3]. Chronic cutaneous lupus erythematosus was reported as first manifestation in up to $4 \%$ cSLE patients and in up to $10 \%$ during the course of disease $[1,3]$.

Lupus erythematosus panniculitis (LEP) is a rare form of chronic cutaneous lupus erythematosus described from 2 to $5 \%$ of adult SLE [4-6]. In CSLE, LEP data are limited to few case reports [7-10].

Therefore, the objective of the present multicenter cohort study was to evaluate the prevalence of LEP and its possible association with demographic data, clinical manifestations, laboratory abnormalities,

(C) The Author(s). 2019 Open Access This article is distributed under the terms of the Creative Commons Attribution 4.0 International License (http://creativecommons.org/licenses/by/4.0/), which permits unrestricted use, distribution, and 
disease activity score, treatment and outcome in a large cSLE population.

\section{Methods}

This study was conducted in 10 pediatric rheumatology services in the state of São Paulo, Brazil including a population of 847 cSLE patients [3]. All patients fulfilled the American College of Rheumatology (ACR) criteria for SLE [11], with disease onset before the age of 18 [12]. This study was approved by the Ethical Committee of University of São Paulo (CAPPESq number 09231912.2.1001.0068) and the consent from the patient for publication of these images was also obtained. The study was also approved by the others University Hospital participating in the present study. An investigator meeting was held for this study in São Paulo city to delineate the protocol according to the clinical parameters definitions and disease activity tool scoring. Investigators in each one of the centers, using the same specific database, conducted data collection locally. One or more rounds of queries were performed to check for accuracy and sort out discrepancies [3, 13].

Patient's medical charts were systematically reviewed according to demographic data, clinical features and LEP characteristics, laboratorial abnormalities, therapeutic data and outcome. LEP was diagnosed by the presence of painful subcutaneous nodules or plaques in deep dermis and subcutaneous tissues. Skin biopsy confirmed lobular or mixed panniculitis with lymphocytic lobular inflammatory infiltrate in all patients [8]. Patients were divided in two groups with similar disease duration: cSLE patients with LEP (evaluated at LEP diagnosis) and cSLE patients without LEP (evaluated at last visit).

Descriptors of SLE Disease Activity Index 2000 (SLEDAI-2 K) were used to define clinical manifestations [14], and custom definitions as previously reported [3, 13]. Constitutional involvement included fever, lymphadenopathy (peripheral lymph node enlargement $>1.0 \mathrm{~cm}$ ), hepatomegaly [based on physical exam with liver edge $\geq 2 \mathrm{~cm}$ below the right costal margin or imaging (ultrasound or computer tomography when available)] and/or splenomegaly [based on physical exam with palpable spleen or imaging (ultrasound or computer tomography when available)] [3]. Neuropsychiatric lupus included 19 syndromes according to ACR classification criteria [15]. Antiphospholipid syndrome (APS) was diagnosed according to the presence of arterial and/or venous thrombosis and antiphospholipid antibodies [16].

Laboratorial assessment included complete blood cell count and urine examination. Anti-double-stranded DNA (anti-dsDNA), anticardiolipin antibodies (aCL) IgG and IgM were carried out at each center and the cutoff values were considered to be valid. Lupus anticoagulant was detected according to the guidelines of the International Society on Thrombosis and Hemostasis [17].

Drug treatment data [prednisone, intravenous methylprednisolone, chloroquine diphosphate, hydroxychloroquine sulfate, methotrexate, azathioprine, cyclosporine, mycophenolate mofetil, intravenous cyclophosphamide (IVCYC), intravenous immunoglobulin (IVIG) and rituximab] were also recorded.

\section{Statistical analysis}

Results were presented as an absolute number (frequency) for categorical variables and median (range) or mean \pm standard deviation for continuous variables. Categorical variables comparisons were assessed by Pearson $X$-Square or Fisher's exact test. Continuous variables from cSLE patients with and without LEP were compared by Mann-Whitney test or t test as appropriate. Statistical analysis was performed using Bonferroni correction $(p<0.004)$.

\section{Results}

LEP was observed in 6/847 (0.7\%) cSLE patients. LEP was the first disease manifestation in 1/6 cSLE patient (2.5 years before cSLE diagnosis), occurred at diagnosis in $2 / 6$ patients and after diagnosis in $3 / 6$ patients (with 5.5, 9.91 and 11.4 years after cSLE diagnosis) (Table 1 ). Painful subcutaneous erythematous and indurated nodules were observed in 6/6 LEP patients, and concomitant painful subcutaneous erythematous plaques in 4/6. Generalized distribution (including face, trunk and limbs) was evidenced in 3/6 LEP patients and it was localized in upper limbs in 2/6 and face in 1/6. Cutaneous hyperpigmentation and/or cutaneous atrophy occurred in 5/6. Histopathology findings showed lobular panniculitis without vasculitis in $5 / 6$ and panniculitis with vasculitis in $1 / 6$ (Table 1 ). One female patient (Case 4) had sepsis and LEP as initial cSLE manifestation and she deceased before lupus diagnosis and prior to immunosuppressive treatment. Her biopsy revealed LEP associated with skin obliterative vasculopathy with positive serology for antiphospholipid antibodies.

Figure 1 shows LEP with cutaneous atrophy affecting the back and limbs. Figure 2 shows details of LEP patient with subcutaneous nodules, cutaneous atrophic and hyperpigmentated skin. Figure 3 shows histopathology of a skin biopsy of a LEP cSLE patient.

Treatment for LEP included: prednisone in 5/6 (83\%), antimalarial drugs in $4 / 6(67 \%)$, methotrexate in $3 / 6(50 \%)$, azathioprine in $2 / 6(33 \%)$ and one patient used cyclosporine. Regarding response for LEP treatment, 3/6 had refractory LEP to glucocorticoid, anti-malarial drugs and immunosuppressive agents (cases 2, 5 and 6). These cSLE patients improved the recurrent painful subcutaneous erythematous nodules and plaques after IVIG (case 2), rituximab (case 5) 
Table 1 Cutaneous manifestations, demographic data, disease activity, outcome, skin biopsy and treatment in six childhood-onset systemic lupus erythematosus (cSLE) patients with lupus erythematosus panniculitis (LEP)

\begin{tabular}{|c|c|c|c|c|c|c|}
\hline Patient & $\begin{array}{l}\text { Interval between LEP } \\
\text { and CSLE diagnosis, } \\
\text { years }\end{array}$ & SLEDAI-2 K & $\begin{array}{l}\text { Painful Plaques / } \\
\text { painful } \\
\text { subcutaneous } \\
\text { nodules }\end{array}$ & $\begin{array}{l}\text { Cutaneous } \\
\text { hyperpigmentation / } \\
\text { hypopigmentation / } \\
\text { atrophy }\end{array}$ & $\begin{array}{l}\text { Skin biopsy findings; direct } \\
\text { immunofluorescence } \\
\text { staining (DIF) }\end{array}$ & Treatments \\
\hline 1 & -2.5 & 3 & $-1+$ & $+1-1+$ & LP; mild GCD of IgM at DEJ) & PD, AM, NSAID, AZA \\
\hline 2 & 11.4 & 13 & $+/+$ & $+/-/+$ & $\begin{array}{l}\text { LP with vasculitis; moderate } \\
\text { GCD of IgM at DEJ) }\end{array}$ & $\begin{array}{l}\text { PD, MP, AM, AZA, } \\
\text { MTX, IVIG }\end{array}$ \\
\hline 3 & 5.5 & 13 & $+/+$ & $+1+1+$ & $\begin{array}{l}\text { LP; intense GCD of } \lg G, \lg G \\
\lg A \text { and } C 3 \text { at } D E J\end{array}$ & $P D, M P, C Y, M T X$ \\
\hline 4 & 0 & 25 & $-1+$ & $-1-1-$ & $\begin{array}{l}\text { LP, vasculopathy obliterans; } \\
\text { absence of IgG, IgM, IgA, C3 } \\
\text { and C4 at DEJ }\end{array}$ & No treatment ${ }^{a}$ \\
\hline 5 & 0 & 0 & $+/+$ & $+1+1+$ & $\begin{array}{l}\text { LP; absence of IgG, IgM, IgA, } \\
\text { C3 and C4 at DEJ }\end{array}$ & $\begin{array}{l}\mathrm{PD}, \mathrm{AM}, \mathrm{NSAID}, \mathrm{MMF}, \\
\text { rituximab }\end{array}$ \\
\hline 6 & 9.91 & 5 & $+/+$ & $+/-1+$ & $\mathrm{LP} ; \mathrm{ND}$ & $\begin{array}{l}\text { PD, MP, AM, MTX, MMF, } \\
\text { IVCYC }\end{array}$ \\
\hline
\end{tabular}

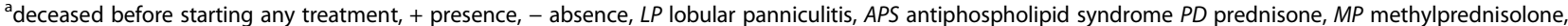
NSAID nonsteroidal anti-inflammatory, $A M$ antimalarial, $A Z A$ azathioprine, IVCYC intravenous cyclophosphamide, $C Y$ cyclosporine, $M T X$ methotrexate, $M M F$ mycopheneolate mofetil, IVIG intravenous immunoglobulin, GCD granular and continuous deposits, DEJ dermo-epidermal junction, ND not done, SLEDAI-2 K Systemic Lupus Erythematosus Disease Activity Index 2000

and IVCYC (case 6). Regarding outcomes, skin hyperpigmentation was observed in 5/6 LEP patients, skin hypopigmentation in $2 / 6$ and cutaneous atrophy in $5 / 6$.

LEP patients $(n=6)$ were compared with 60 patients without LEP (ratio of 1:10). These 60 patients without LEP were randomly selected of $841 \mathrm{cSLE}$ patients without LEP and that presented same disease duration of LEP patients. Further comparison between 6 cSLE patients with LEP compared to 60 cSLE patients without LEP with the same disease duration [2.75 (0-11.4) vs. $2.83(0-11.8)$ years, $p=0.297]$, showed higher frequencies of constitutional involvement $(67 \%$ vs. $10 \%, p=0.003)$ and leukopenia (67\% vs. $7 \%, p=0.002)$ in the former group. The median of serum CRP values was significantly higher in cSLE patients with LEP compared to cSLE control patients without LEP (10.5 vs. $0.5 \mathrm{mg} / \mathrm{L}, p=0.001)$.

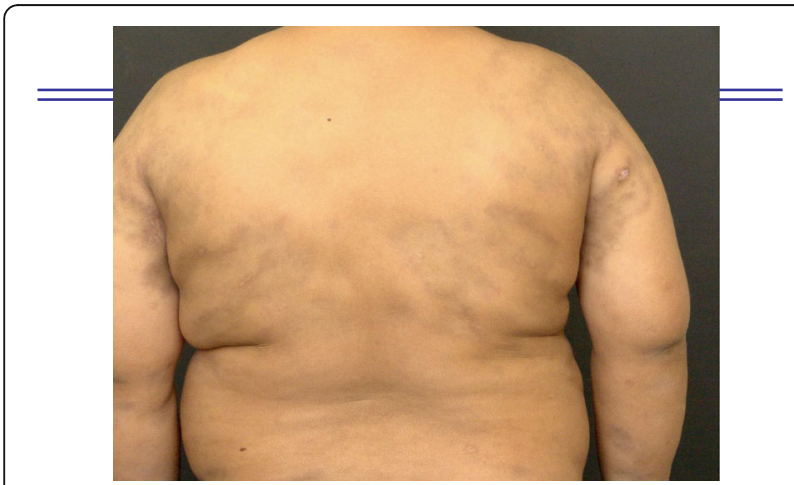

Fig. 1 Lupus erythematosus panniculitis showing extense areas of cutaneous atrophy affecting the back and limbs.
Frequencies of major organ involvements such as renal and neuropsychiatric were similar in both groups $(p>0.004)$. The median of SLEDAI-2 K was similar in the two groups [9 (0$25)$ vs. 4 (0-41), $p=0.352$ ] (Table 2 ).

\section{Discussion}

This was the first study to assess panniculitis in a large cSLE population. LEP was a rare skin manifestation of cSLE occurring mainly in the first three years of disease.

The multicentric design with a large cohort of pediatric patients allowed a more precise evaluation of this rare lupus manifestation. All cSLE patients with suspect of LESP were included in the present study and the diagnoses were confirmed according to the histopathology

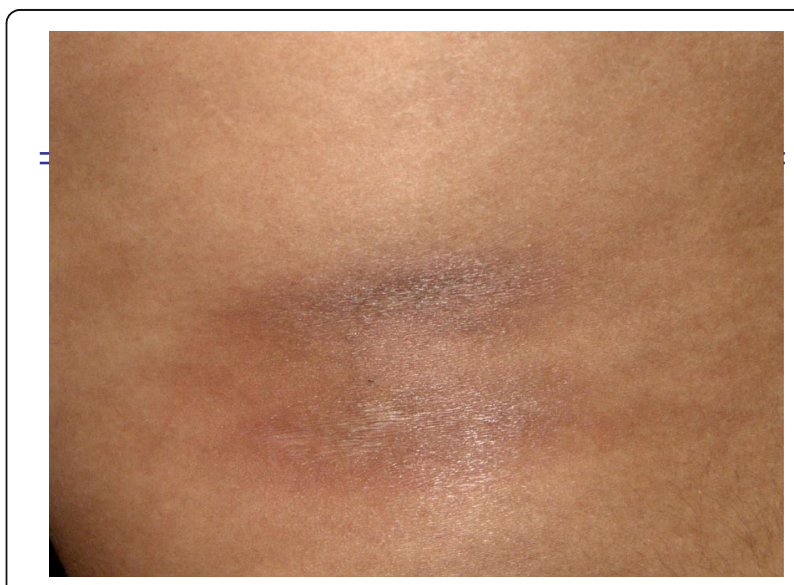

Fig. 2 Detail of lupus panniculitis presenting subcutaneous nodules with cutaneous atrophic and hyperpigmentated skin. 


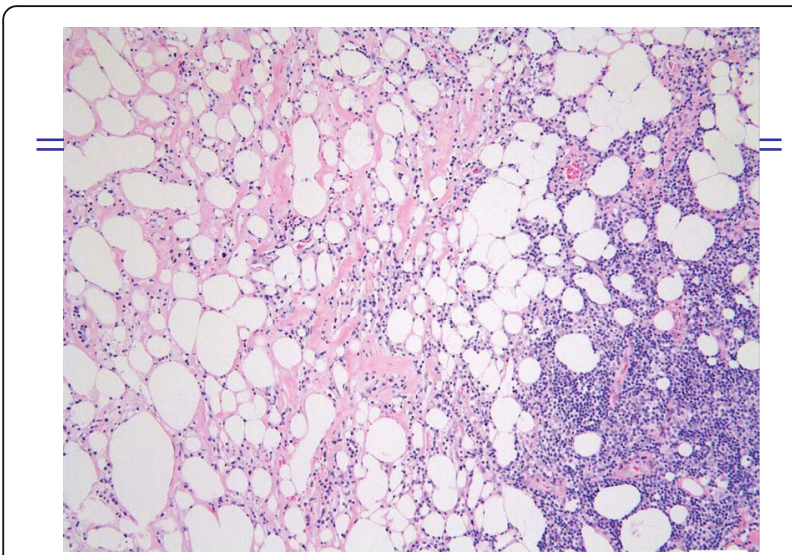

Fig. 3 Histopathology of a skin biopsy of the arm presenting septal and lobular panniculitis, with inflammatory infiltrate predominantly composed of lymphocytes in fat lobules and hyaline fat necrosis

findings. The limitation was the retrospective analysis with potential missing data and for this reason an investigator meeting was performed to standardize the protocol study in all centers involved. The low number of cSLE patients with LEP observed herein was also the limitation of the present study. However to minimize bias, the comparisons of clinical manifestations, laboratorial abnormalities and treatments were performed in both groups assessing same disease duration, with a ratio of 1:10 (1 CSLE with LEP, 10 cSLE without LEP patients).
Importantly, the diagnosis of panniculitis in all patients was based not only on the typical clinical manifestations with nodules and/or plaques in deep dermis and subcutaneous adipose tissues, but it was also confirmed through histopathological features [18]. In fact, skin biopsy is essential to exclude other causes of inflammation of the fatty tissue, such as lymphoma, deep morphea, erythema nodosum and sarcoidosis [18]. Lymphocytic vasculitis may also be an additional and typical cutaneous histopathology abnormality in LEP patients $[4,5,19]$. Interestingly one of our cSLE patients had LEP associated with vasculopathy obliterans probably due to APS, as also described in two adult SLE [20].

We confirmed previous observation that LEP in cSLE patients has a predilection for face [4] and we described that in half of our cases the distribution of skin lesions were generalized, a condition reported to be extremely rare [21].

In our study children and adolescents with lupus panniculitis had a mild systemic disease, characterized by constitutional involvement and leukopenia. Indeed other studies observed that this cutaneous chronic manifestation might be an indicator of a less severe systemic lupus [4, 9, 22-24].

Treatment for cSLE includes corticosteroids, antimalarial and immunosuppressive drugs $[25,26]$, as also prescribed for LEP patients evaluated in this study. Depressed lipoatrophic areas were very frequent

Table 2 Demographic data, cumulative clinical manifestations and laboratory parameters, and SLEDAl-2 K in childhood-onset systemic lupus erythematosous (CSLE) patients with lupus erythematosus panniculitis (LEP)

\begin{tabular}{|c|c|c|c|}
\hline Variables & CSLE with LEP (at diagnosis) $(n=6)$ & CSLE without LEP (at last visit) $(n=60)$ & $P$ \\
\hline \multicolumn{4}{|l|}{ Demographic data } \\
\hline Age at diagnosis, years & $15(10-21)$ & $15(2-25)$ & 0.624 \\
\hline Female gender & $4(67)$ & $52(87)$ & 0.222 \\
\hline Disease duration, years & $2.75(0-11.4)$ & $2.83(0-11.8)$ & 0.297 \\
\hline \multicolumn{4}{|l|}{ Cumulative clinical manifestations } \\
\hline Constitutional involvement & $4(67)$ & $6(10)$ & 0.003 \\
\hline Cutaneous vasculitis & $3(50)$ & $6(10)$ & 0.029 \\
\hline Musculoskeletal involvement & $3(50)$ & $4(7)$ & 0.013 \\
\hline Serositis & $0(0)$ & $4(7)$ & 1.000 \\
\hline Neuropsychiatric involvement & $0(0)$ & $8(13)$ & 1.000 \\
\hline Nephritis & $1(17)$ & $19 / 59(32)$ & 0.657 \\
\hline \multicolumn{4}{|l|}{ Cumulative laboratory parameters } \\
\hline Leukopenia, $<4000 / \mathrm{mm}^{3}$ & $4(67)$ & $4 / 55(7)$ & 0.002 \\
\hline Anti-ds-DNA & $4(67)$ & $17 / 47(36)$ & 0.200 \\
\hline \multicolumn{4}{|l|}{ Disease activity at diagnosis } \\
\hline SLEDAI-2 K & $9(0-25)$ & $4(0-41)$ & 0.352 \\
\hline
\end{tabular}

* $P$ - value according to Bonferroni correction for multiple comparisons $(p<0.004)$. Results are presented in $\mathrm{n}(\%)$ and median (range), SLEDAl-2 K - Systemic Lupus Erythematosus Disease Activity Index 2000 
sequelae at cSLE diagnosis, reinforcing the concept that this residual scarring induces great morbidity associated with cosmetic abnormalities [4]. Hyperpigmentation and hypopigmentation are described in these patients and the former was more often observed in our patients $[4,5,8,9,27]$.

\section{Conclusion}

Panniculitis is a rare skin manifestation of cSLE occurring in the first three years of disease with a high frequency of sequelae. The majority of patients presented concomitant mild lupus manifestations.

\section{Funding}

This study was supported by grants from Conselho Nacional de Desenvolvimento Científico e Tecnológico (CNPq 301805/2013-0 to RMRP, $303752 / 2015-7$ to MTT, 301479/2015-1 to CSM, 305068/2014-8 to EB and $303422 / 2015-7$ to CAS), Federico Foundation (to EB, RMRP and CAS) and by Núcleo de Apoio à Pesquisa "Saúde da Criança e do Adolescente" da USP (NAP-CriAd) to CAS.

\section{Availability of data and materials}

Not applicable.

\section{Authors' contributions}

All authors analyzed and interpreted the patient data regarding autoimmune hepatitis in childhood onset systemic lupus erythematosus. MV, PA, NWSG, $\mathrm{EB}$ and $\mathrm{CAS}$ were the major contributor in writing the manuscript. All authors read and approved the final manuscript.

\section{Ethics approval and consent to participate}

This study was approved by our Ethics Committee.

\section{Consent for publication}

The patient provided consent for publication of the images.

\section{Competing interests}

Not applicable.

\section{Publisher's Note}

Springer Nature remains neutral with regard to jurisdictional claims in published maps and institutional affiliations.

\section{Author details}

'Pediatric Rheumatology Unit, Children's Institute, Hospital das Clinicas HCFMUSP, Faculdade de Medicina, Universidade de Sao Paulo, Sao Paulo, SP, BR, Brazil. ${ }^{2}$ Division of Rheumatology, Hospital das Clinicas HCFMUSP, Faculdade de Medicina, Universidade de Sao Paulo, Av. Dr. Eneas Carvalho Aguiar, 647 - Cerqueira César, São Paulo, SP 05403-000, Brazil. ${ }^{3}$ Division of Dermatology, Hospital das Clinicas HCFMUSP, Faculdade de Medicina, Universidade de Sao Paulo, Sao Paulo, SP, BR, Brazil. ${ }^{4}$ Pediatric Rheumatology Unit, Universidade Federal de São Paulo, São Paulo, Brazil. ${ }^{5}$ São Paulo State University (UNESP) - Faculdade de Medicina de Botucatu, Botucatu, Brazil. ${ }^{6}$ Pediatric Rheumatology Unit, Ribeirão Preto Medical School - University of

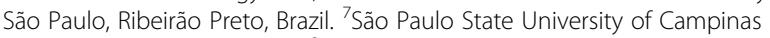
(UNICAMP), Campinas, Brazil. ${ }^{8}$ Irmandade da Santa Casa de Misericórdia de São Paulo, São Paulo, Brazil.

Received: 28 September 2018 Accepted: 9 January 2019

Published online: 18 January 2019

\section{References}

1. Silva CA. Childhood-onset systemic lupus erythematosus: early disease manifestations that the paediatrician must know. Expert Rev Clin Immunol. 2016;12:907-10.
2. Chiewchengchol D, Murphy R, Edwards SW, Beresford MW. Mucocutaneous manifestations in juvenile-onset systemic lupus erythematosus: a review of literature. Pediatr Rheumatol Online J. 2015;13:1.

3. Gomes RC, Silva MF, Kozu K, Bonfá E, Pereira RM, Terreri MT, et al. Features of 847 childhood-onset systemic lupus Erythematousus patients in three age groups at diagnosis: a Brazilian multicenter study. Arthritis Care Res. 2016:68:1736-41.

4. Fraga J, García-Díez A. Lupus erythematosus panniculitis. Dermatol Clin. 2008:26:453-63.

5. Park HS, Choi JW, Kim BK, Cho KH. Lupus erythematosus panniculitis: clinicopathological, immunophenotypic, and molecular studies. Am J Dermatopathol. 2010;32:24-30.

6. Bednarek A, Bartoszak L, Samborski W. Case report on a patient with lupus panniculitis. Postepy Dermatol Alergol. 2015;32:59-62.

7. Fernandes S, Santos S, Freitas I, Salgado M, Afonso A, Cardoso J. Linear lupus erythematosus profundus as an initial manifestation of systemic lupus erythematosus in a child. Pediatr Dermatol. 2014;31:378-80.

8. Guissa VR, Trudes G, Jesus AA, Aikawa NE, Romiti R, Silva CA. Lupus erythematosus panniculitis in children and adolescents. Acta Reumatol Port. 2012;37:82-5.

9. Weingartner JS, Zedek DC, Burkhart CN, Morrell DS. Lupus erythematosus panniculitis in children: report of three cases and review of previously reported cases. Pediatr Dermatol. 2012;29:169-76.

10. Wimmershoff MB, Hohenleutner U, Landthaler M. Discoid lupus erythematosus and lupus profundus in childhood: a report of two cases. Pediatr Dermatol. 2003;20:140-5.

11. Hochberg MC. Updating the American College of Rheumatology revised criteria for the classification of systemic lupus erhytematosus. Arthritis Rheum. 1997:40:1725.

12. Mina R, Brunner HI. Update on differences between childhood-onset and adult-onset systemic lupus erythematosus. Arthritis Res Ther. 2013;15:218.

13. Marques VL, Gormezano NW, Bonfá E, Aikawa NE, Terreri MT, Pereira RM, et al. Pancreatitis subtypes survey in 852 childhood-onset systemic lupus erythematosus patients. J Pediatr Gastroenterol Nutr. 2016:62:328-34.

14. Gladman DD, Ibañez D, Urowitz MB. Systemic lupus erythematosus disease activity index 2000. J Rheumatol. 2002;29:288-91.

15. American College of Rheumatology Ad Hoc committee on neuropsychiatric Lupus Syndromes. The American College of Rheumatology nomenclature and case definitions for neuropsychiatric lupus syndromes. Arthritis Rheum. 1999:42:599-608.

16. Avcin T, Cimaz R, Rozman B. The Ped-APS Registry: the antiphospholipid syndrome in childhood. Lupus. 2009;18:894-9.

17. Brandt JT, Triplett DA, Alving B, Scharrer I. Criteria for the diagnosis of lupus anticoagulants: an update. On behalf of the subcommittee on lupus anticoagulant/antiphospholipid antibody of the scientific and standardisation committee of the ISTH. Thromb Haemost. 1995;74:1185-90.

18. Moraes AJ, Soares PM, Zapata AL, Lotito AP, Sallum AM, Silva CA. Panniculitis in childhood and adolescence. Pediatr Int. 2006;48:48-53.

19. Chopra R, Chhabra S, Thami GP, Punia RP. Panniculitis: clinical overlap and the significance of biopsy findings. J Cutan Pathol. 2010;37:49-58.

20. Arai $\mathrm{S}$, Katsuoka K, Eto H. An unusual form of lupus erythematosus profundus associated with antiphospholipid syndrome: report of two cases. Acta Derm Venereol. 2013;93:581-2.

21. Nousari HC, Kimyai-Asadi A, Provost TT. Generalized lupus erythematosus profundus in a patient with genetic partial deficiency of C4. J Am Acad Dermatol. 1999:41:362-4.

22. Martens PB, Moder KG, Ahmed I. Lupus panniculitis: clinical perspectives from a case series. J Rheumatol. 1999;26:68-72.

23. Koransky JS, Esterly NB. Lupus panniculitis (profundus). J Pediatr. 1981;98:241-4.

24. Taïeb A, Hehunstre JP, Goetz J, Surlève Bazeille JE, Fizet D, Hauptmann G, et al. Lupus erythematosus panniculitis with partial genetic deficiency of C2 and C4 in a child. Arch Dermatol. 1986:122:576-82.

25. Silva CA, Aikawa NE, Pereira RM, Campos LM. Management considerations for childhood-onset systemic lupus erythematosus patients and implications on therapy. Expert Rev Clin Immunol. 2016;12:301-13.

26. Klumb EM, Silva CA, Lanna CC, Sato El, Borba EF, Brenol JC, et al. Consensus of the Brazilian Society of Rheumatology for the diagnosis, management and treatment of lupus nephritis. Rev Bras Reumatol. 2015;55:1-21.

27. Vera-Recabarren MA, García-Carrasco M, Ramos-Casals M, Herrero C. Comparative analysis of subacute cutaneous lupus erythematosus and chronic cutaneous lupus erythematosus: clinical and immunological study of 270 patients. Br J Dermatol. 2010;162:91-101. 